International Journal of Pure and Applied Mathematics

Volume 112 No. 1 2017, 145-157

ISSN: 1311-8080 (printed version); ISSN: 1314-3395 (on-line version)

url: http://www.ijpam.eu

doi: 10.12732/ijpam.v112i1.12

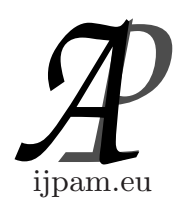

\title{
IMPLICATION-BASED T-FUZZY SUBGROUP OF \\ A FINITE GROUP AND ITS PROPERTIES
}

\author{
M. Selva Rathi ${ }^{1 \S}$, J. Michael Anna Spinneli ${ }^{2}$ \\ ${ }^{1}$ Department of Mathematics \\ Karunya University \\ Coimbatore, INDIA \\ ${ }^{2}$ Department of Mathematics
}

L.R.G. Government Arts College for Women

Tirupur, INDIA

\begin{abstract}
Based on the definition of implication-based fuzzy subgroup of a finite group given by Yuan, implication-based T-fuzzy subgroup and implication-based T-normal fuzzy subgroup of a finite group is defined. Some properties of them are proved in this paper. Also Tproduct, $\dot{T}$ - product and $T^{\prime}$ - product of these implication-based T-fuzzy subgroups of a finite group are defined and its properties are discussed.
\end{abstract}

AMS Subject Classification: 03E72, 08A72, 20N25

Key Words: implication-based fuzzy subgroup, implication-based T-fuzzy subgroup, implication-based T-fuzzy normal subgroup

\section{Introduction}

In 1965 the concept of fuzzy set was first introduced by Zadeh [1]. Rosenfeld [2] and many others [3], [4], [5] have studied about the fuzzy normal subgroup. Anthony and Sherwood [6] redefined fuzzy subgroups using t-norm. Many including Sessa [7] studied about these T-fuzzy subgroups and proved many prop-

Received: $\quad$ September 29, 2016

Revised: December 2, 2016

Published: January 25, 2017

(c) 2017 Academic Publications, Ltd. url: www.acadpubl.eu

$\S_{\text {Correspondence author }}$ 
erties. Yuan [8] defined implication-based fuzzy subgroup in 2003. In this paper we define the concept of implication-based T-fuzzy subgroup of a finite group and its properties. We also define the $T$ - product, $\dot{T}-$ product and $T^{\prime}-$ product of these implication-based T-fuzzy subgroups of a finite group and proved some properties.

\section{Preliminaries}

Let $X$ be an universe of discourse and $(G, \cdot)$ be a group. In fuzzy logic, truth value of fuzzy proposition $\alpha$ is denoted by $[\alpha]$. The fuzzy logical and the corresponding set theoretical notations used in this paper are:

1. $(x \in A)=A(x)$;

2. $(\alpha \wedge \beta)=\min \{[\alpha],[\beta]\}$

3. $(\alpha \rightarrow \beta)=\min \{1,1-[\alpha]+[\beta]\}$;

4. $(\forall x \alpha(x))=i n f_{x \in X}[\alpha(x)]$;

5. $(\exists x \alpha(x))=\sup _{x \in X}[\alpha(x)]$;

$\vDash \alpha \Leftrightarrow[\alpha]=1$ for all valuations. The truth valuation rules given above are those in Lukasiewicz system of continuous-valued logic.

Definition 1. [7] A triangular norm is a real continuous function $t$ : $[0,1] \times[0,1] \rightarrow[0,1]$ fulfilling the following properties, for every $a, b, c, d \in[0,1]$

(i) $t(0, a)=0, t(a, 1)=a$ [boundary conditions],

(ii) $t(a, b) \leq t(c, d)$ if $a \leq c$ and $b \leq d$ [monotonicity],

(iii) $t(a, b)=t(b, a)[$ commutativity],

(iv) $t(t(a, b), c)=t(a, t(b, c))$ [associativity]

Definition 2. [8] If a fuzzy subset $A$ of a group $G$ satisfies for any $x, y \in G$

(i) $\vDash(x \in A) \wedge(y \in A) \rightarrow(x y \in A)$

(ii) $\vDash(x \in A) \rightarrow\left(x^{-1} \in A\right)$

Then $A$ is called a fuzzifying subgroup. 
Definition 3. [8] Let $A$ be a fuzzy subset of a finite group $G$ and $\lambda \in(0,1]$ is a fixed number. If for any $x, y \in G$

(i) $\vDash_{\lambda}(x \in A) \wedge(y \in A) \rightarrow(x y \in A)$

(ii) $\vDash_{\lambda}(x \in A) \rightarrow\left(x^{-1} \in A\right)$

Then $A$ is called an implication-based fuzzy subgroup of $G$.

Definition 4. [9] Let $A$ be an implication-based fuzzy subgroup of $G$ and $f: G \rightarrow G$ be a function defined on $G$. Then the implication-based fuzzy subgroup $B$ of $f(G)$ is defined by

$$
\begin{gathered}
\vDash_{\lambda}\left(\exists x\{(x \in A)\} ; x \in f^{-1}(y)\right) \rightarrow \\
(y \in B),
\end{gathered}
$$

for all $y \in f(G)$.

Similarly if $B$ is an implication-based fuzzy subgroup of $f(G)$ then the implication-based fuzzy subgroup $A=f \circ B$ in $G$ is defined as $\vDash_{\lambda}(f(x) \in$ $B) \rightarrow(x \in A)$ for all $x \in G$ and is called the pre-image of $B$ under $f$.

Definition 5. [9] An implication-based fuzzy subgroup $A$ of $G$ is called an implication-based fuzzy normal subgroup if $\vDash_{\lambda}(x y \in A) \rightarrow(y x \in A) \quad \forall x, y \in G$

Lemma 1. [9] An homomorphic image or pre-image of an implicationbased fuzzy subgroup is an implication-based fuzzy subgroup provided in the former case the sup-property holds.

Lemma 2. [10] Generalised Associative Law Let $T:[0,1] \times[0,1] \rightarrow[0,1]$ be a t-norm then $T((T(a, b)),(T(c, d)))=T((T(a, c)),(T(b, d))) \quad \forall a, b, c, d \in$ $[0,1]$

Proof. Let $a, b, c, d \in[0,1]$. Then

$$
\begin{aligned}
T((T(a, b)),(T(c, d))) & =T(a, T(b, T(c, d))) \quad \text { by (iii) and (iv) } \\
& =T(a, T(T(b, c), d)) \\
& =T(a, T(T(c, b), d)) \\
& =T(a, T(c, T(b, d))) \\
& =T(T(a, c), T(b, d)) .
\end{aligned}
$$

Definition 6. [11] Given two t-norms $T_{1}$ and $T_{2}, T_{1}$ is said to be stronger than $T_{2}$, if $T_{1}(x, y) \geq T_{2}(x, y) \quad \forall x, y \in[0,1]$ and is written as $T_{1} \geq T_{2}$. And $T_{1}$ is said to dominate $T_{2}$, if $T_{1}\left(\left(T_{2}(a, b)\right),\left(T_{2}(c, d)\right)\right) \geq T_{2}\left(\left(T_{1}(a, c)\right),\left(T_{1}(b, d)\right)\right)$ $\forall a, b, c, d \in[0,1]$ and is written as $T_{1} \gg T_{2}$. 
Let $(G, \cdot)$ be a finite group with the identity element ' $e^{\prime}, \lambda \in(0,1]$ be a fixed number and let $T:[0,1] \times[0,1] \rightarrow[0,1]$ be a $t-$ norm.

\section{Implication-Based T-Fuzzy Subgroup and its Properties}

Definition 7. Let $A$ be a fuzzy subset of $\mathrm{G}$. For any $x, y \in G$ if

$$
\vDash_{\lambda}(T((x \in A),(y \in A))) \rightarrow(x y \in A)
$$

Then $A$ is called an implication-based $T$ - fuzzy subgroupoid of $\mathrm{G}$.

Definition 8. Let $A$ be a fuzzy subset of G. For any $x, y \in G$ if (i) $\vDash_{\lambda}(T((x \in A),(y \in A))) \rightarrow(x y \in A)$

(ii) $\vDash_{\lambda}(x \in A) \rightarrow\left(x^{-1} \in A\right)$

Then $A$ is called an implication-based T-fuzzy subgroup of $\mathrm{G}$.

Example for implication-based T-fuzzy subgroup of a finite group.

Consider the group $G=\{e, a, b, c\}$ along with the binary operation ' $*$ ' whose closure table is as follows.

\begin{tabular}{|c|c|c|c|c|}
\hline$*$ & $\mathrm{e}$ & $\mathrm{a}$ & $\mathrm{b}$ & $\mathrm{c}$ \\
\hline $\mathrm{e}$ & $\mathrm{e}$ & $\mathrm{a}$ & $\mathrm{b}$ & $\mathrm{c}$ \\
\hline $\mathrm{a}$ & $\mathrm{a}$ & $\mathrm{e}$ & $\mathrm{c}$ & $\mathrm{b}$ \\
\hline $\mathrm{b}$ & $\mathrm{b}$ & $\mathrm{c}$ & $\mathrm{e}$ & $\mathrm{a}$ \\
\hline $\mathrm{c}$ & $\mathrm{c}$ & $\mathrm{b}$ & $\mathrm{a}$ & $\mathrm{e}$ \\
\hline
\end{tabular}

For the fuzzy set $A: G \rightarrow[0,1]$ defined by $A(e)=1, A(a)=.25, A(b)=$ $.5, A(c)=.75$ with $\lambda=.2$ and the implication operator is that of Lukasiewicz, with the t-norm defined by $T(a, b)=a b$ we have

\begin{tabular}{|c|c|c|c|c|}
\hline $\mathrm{T}$ & {$[e]$} & {$[a]$} & {$[b]$} & {$[c]$} \\
\hline$[e]$ & 1 & .25 & .5 & .75 \\
\hline$[a]$ & .25 & .0625 & .125 & .1875 \\
\hline$[b]$ & .5 & .125 & .25 & .375 \\
\hline$[c]$ & .75 & .1875 & .375 & .5625 \\
\hline
\end{tabular}

Then $A$ is an implication-based T-fuzzy subgroup of $G$.

Theorem 3. Let $f$ be a homomorphism of the group $G$ and $B$ be an implication-based T-fuzzy subgroup of $f(G)$ then $A=f \circ B$ is an implicationbased $T$-fuzzy subgroup of $G$. 
Proof. Let $x, y \in G$

(i)

$\vDash_{\lambda}(T((x \in A),(y \in A)))$

$\rightarrow(T((f(x) \in B),(f(y) \in B)))$

$\rightarrow(f(x) f(y) \in B) \because B$ is an implication-based T-fuzzy subgroup of $G$

$\rightarrow(f(x y) \in B) \because f$ is an homomorphism

$\rightarrow(x y \in A)$

(ii) $\vDash_{\lambda}(x \in A) \rightarrow\left(x^{-1} \in A\right) \forall x \in G$ follows from lemma 1

$\therefore A=f \circ B$ is an implication-based T-fuzzy subgroup of $G$.

Theorem 4. Let $A$ be an implication-based T-fuzzy subgroup of $G$ and $f$ be an homomorphism on $G$. Then $B$ the image of $A$ under $f$ is also an implication-based T-fuzzy subgroup of $f(G)$.

Proof. (i) Let $y_{1}, y_{2} \in f(G)$.

Let $A_{1}=f^{-1}\left(y_{1}\right)$,

$A_{2}=f^{-1}\left(y_{2}\right)$,

$A_{12}=f^{-1}\left(y_{1} y_{2}\right)$

$A_{1} A_{2}=\left\{x \in G / x=a_{1} a_{2}\right.$ for some $\left.a_{1} \in A_{1}, a_{2} \in A_{2}\right\}$.

Then $x \in G$

$\vDash_{\lambda}\left(x \in A_{1} A_{2}\right) \rightarrow\left(a_{1} a_{2} \in A_{1} A_{2}\right)$

where $a_{1} \in A_{1}, a_{2} \in A_{2}$

$\rightarrow\left(f\left(a_{1} a_{2}\right) \in f\left(A_{1} A_{2}\right)\right)$

$\rightarrow\left(f\left(a_{1}\right) f\left(a_{2}\right)=y_{1} y_{2} \in f\left(A_{12}\right)\right)$

$\rightarrow\left(y_{1} y_{2} \in f\left(A_{12}\right)\right)$

$\rightarrow\left(f(x) \in f\left(A_{12}\right)\right)$

$\rightarrow\left(x \in A_{12}\right)$

$\therefore A_{1} A_{2} \leq A_{12}$

Now

$\vDash_{\lambda}\left(\exists x_{1} x_{2}\left\{T\left(\left(x_{1} \in A\right),\left(x_{2} \in A\right)\right)\right\}\right.$;

$\left.x_{1} \in A_{1}, x_{2} \in A_{2}\right)$

$\rightarrow\left(\exists x_{1} x_{2}\left\{\left(x_{1} x_{2} \in A\right)\right\} ; x_{1} \in A_{1}, x_{2} \in A_{2}\right)$

$\because \mathrm{A}$ is an implication-based T-fuzzy subgroupoid of $\mathrm{G}$

$\rightarrow\left(\exists x\left\{\left(x \in A_{1} A_{2}\right)\right\} ; x \in A_{1} A_{2}\right)$

$\rightarrow\left(\exists x\left\{\left(x \in A_{12}\right)\right\} ; x \in A_{12}\right)$

$\rightarrow\left(\exists x\left\{\left(x \in A_{12}\right)\right\} ; x \in f^{-1}\left(y_{1} y_{2}\right)\right)$

$\rightarrow\left(y_{1} y_{2} \in B\right)$ 
Since $\mathrm{T}$ is a continuous t-norm, for every $\epsilon>0$ there exists a $\delta>0$ such that

$\vDash_{\lambda}\left(\left(\exists x_{1}\left\{\left(x_{1} \in A\right)\right\} ; x_{1} \in A_{1}\right)-\delta\right) \rightarrow x_{1}^{*}$

$\vDash_{\lambda}\left(\left(\exists x_{2}\left\{\left(x_{2} \in A\right)\right\} ; x_{2} \in A_{2}\right)-\delta\right) \rightarrow x_{2}^{*}$

$\therefore \vDash_{\lambda}\left(T\left(\left(\exists x_{1}\left\{\left(x_{1} \in A\right)\right\} ; x_{1} \in A_{1}\right)\right.\right.$,

$$
\left.\left.\left(\exists x_{2}\left\{\left(x_{2} \in A\right)\right\} ; x_{2} \in A_{2}\right)\right)-\epsilon\right) \rightarrow T\left(x_{1}^{*}, x_{2}^{*}\right)
$$

Choose $a_{1} \in A_{1}$ and $a_{2} \in A_{2}$ such that

$\vDash_{\lambda}\left(\left(\exists x_{1}\left\{\left(x_{1} \in A\right)\right\} ; x_{1} \in A_{1}\right)-\delta\right) \rightarrow\left(a_{1} \in A_{1}\right)$

$\vDash_{\lambda}\left(\left(\exists x_{2}\left\{\left(x_{2} \in A\right)\right\} ; x_{2} \in A_{2}\right)-\delta\right) \rightarrow\left(a_{2} \in A_{2}\right)$

(1) implies

$\vDash_{\lambda}\left(T\left(\left(\exists x_{1}\left\{\left(x_{1} \in A\right)\right\} ; x_{1} \in A_{1}\right),\left(\exists x_{2}\left\{\left(x_{2} \in A\right)\right\} ; x_{2} \in A_{2}\right)\right)-\epsilon\right)$

$$
\rightarrow T\left(\left(a_{1} \in A_{1}\right),\left(a_{2} \in A_{2}\right)\right)
$$

Now $\vDash_{\lambda}\left(T\left(\left(y_{1} \in B\right),\left(y_{2} \in B\right)\right)\right)$

$\rightarrow\left(T\left(\left(\exists x_{1}\left\{\left(x_{1} \in A\right)\right\} ; x_{1} \in A_{1}\right),\left(\exists x_{2}\left\{\left(x_{2} \in A\right)\right\} ; x_{2} \in A_{2}\right)\right)\right)$

$\rightarrow\left(\exists x_{1} x_{2}\left\{T\left(\left(x_{1} \in A\right),\left(x_{2} \in A\right)\right)\right\} ; x_{1} \in A_{1}, x_{2} \in A_{2}\right)$

$\rightarrow\left(\exists x_{1} x_{2}\left\{\left(x_{1} x_{2} \in A\right)\right\} ; x_{1} \in A_{1}, x_{2} \in A_{2}\right)$

$\rightarrow\left(y_{1} y_{2} \in B\right)$

(ii) $\vDash_{\lambda}(x \in A) \rightarrow\left(x^{-1} \in A\right) \forall x \in G$ follows from lemma 1.

$\therefore B$ is an implication-based T-fuzzy subgroupof $f(G)$.

Definition 9. An implication-based T-fuzzy subgroup $A$ of $G$ is called an implication-based T-fuzzy normal subgroup if $\vDash_{\lambda}(x y \in A) \rightarrow(y x \in A) \quad \forall x, y \in G$

Theorem 5. Let $A$ be an implication-based T-fuzzy normal subgroup of $G$ such that $\vDash_{\lambda}(e \in A) \rightarrow 1$ then $B=\left\{x \in G / \vDash_{\lambda}(x \in A) \rightarrow(e \in A)\right\}$ is either empty or a normal subgroup of $G$.

Proof. Let $x, y \in B$. Then

$\vDash_{\lambda}(x \in A) \rightarrow(e \in A)$ and

$\vDash_{\lambda}(y \in A) \rightarrow(e \in A)$.

By the boundary conditions of the t-norm $\mathrm{T}$, we have

$T(a, 1)=1 \Rightarrow T(1,1)=1$.

$\therefore \vDash_{\lambda}(T(1,1))$

$\rightarrow(T((x \in A),(y \in A)))$

$\rightarrow\left(T\left((x \in A),\left(y^{-1} \in A\right)\right)\right)$

$\rightarrow\left(x y^{-1} \in A\right)$

$\therefore \quad \vDash_{\lambda}\left(x y^{-1} \in A\right) \rightarrow(e \in A)$

$\Rightarrow x y^{-1} \in B$. 
Thus $B$ is a subgroup of $G$.

Now let $g \in G, h \in B$

$$
\vDash_{\lambda}\left(g h g^{-1} \in A\right) \rightarrow\left(h g g^{-1} \in A\right)
$$

$\because \mathrm{A}$ is an implication-based T-fuzzy normal subgroup of $\mathrm{G}$

$\rightarrow(h \in A)$

$\rightarrow(e \in A)$

$\therefore g h g^{-1} \in B$

$\Rightarrow B$ is a normal subgroup of $G$.

Theorem 6. Let $A$ be an implication-based T-fuzzy subgroup of $G$ and if there is a sequence $\left\{x_{n}\right\}$ in $G$ such that

$\lim _{n \rightarrow \infty} \vDash_{\lambda}\left(T\left(\left(x_{n} \in A\right),\left(x_{n} \in A\right)\right)\right) \rightarrow 1$

then $\vDash_{\lambda}(e \in A) \rightarrow 1$

Proof. Let $x \in G$

$\vDash_{\lambda}(T((x \in A),(x \in A)))$

$\rightarrow\left(T\left((x \in A),\left(x^{-1} \in A\right)\right)\right)$

$\rightarrow\left(x x^{-1} \in A\right)$

$\rightarrow(e \in A)$

Therefore for each $n$,

$\vDash_{\lambda}\left(T\left(\left(x_{n} \in A\right),\left(x_{n} \in A\right)\right)\right) \rightarrow(e \in A)$

$\lim _{n \rightarrow \infty} \vDash_{\lambda}\left(T\left(\left(x_{n} \in A\right),\left(x_{n} \in A\right)\right)\right)$

$$
\rightarrow(e \in A)
$$

By assumption,

$\lim _{n \rightarrow \infty} \vDash_{\lambda}\left(T\left(\left(x_{n} \in A\right),\left(x_{n} \in A\right)\right)\right) \rightarrow 1$

$\therefore \vDash_{\lambda}(e \in A) \rightarrow 1$

Theorem 7. Let $A$ be an implication-based T-fuzzy subgroup of $G$.

If $\vDash_{\lambda}\left(x y^{-1} \in A\right) \rightarrow 1$ then

(i) $\vDash_{\lambda}(x \in A) \rightarrow(y \in A)$

(ii) $\vDash_{\lambda}(y \in A) \rightarrow(x \in A) \quad \forall x, y \in G$.

Proof. Let $x, y \in G$

$$
\begin{aligned}
\vDash_{\lambda}(x \in A) & \rightarrow\left(x^{-1} \in A\right) \\
& \rightarrow\left(T\left(\left(x^{-1} \in A\right), 1\right)\right) \\
& \rightarrow\left(T\left(\left(x^{-1} \in A\right),\left(x y^{-1} \in A\right)\right)\right) \\
& \rightarrow\left(x^{-1} x y^{-1} \in A\right) \\
& \rightarrow\left(y^{-1} \in A\right) \\
& \rightarrow(y \in A)
\end{aligned}
$$




$$
\begin{aligned}
\vDash_{\lambda}(y \in A) & \rightarrow(T(1,(y \in A))) \\
& \rightarrow\left(T\left(\left(x y^{-1} \in A\right),(y \in A)\right)\right) \\
& \rightarrow\left(x y^{-1} y \in A\right) \\
& \rightarrow(x \in A)
\end{aligned}
$$

Theorem 8. Let $A$ be a fuzzy subset of $G$ and $T$ be a t-norm. If $\vDash_{\lambda}(e \in$ $A) \rightarrow 1$ and $\vDash_{\lambda}(T((x \in A),(y \in A))) \rightarrow\left(x y^{-1} \in A\right) \quad \forall x, y \in G$ then $A$ is an implication-based T-fuzzy subgroup of $G$.

Proof. Let $x, y \in G$

$$
\begin{aligned}
\vDash_{\lambda}(y \in A) & \rightarrow(T(1,(y \in A))) \\
& \rightarrow(T((e \in A),(y \in A))) \\
& \rightarrow\left(e y^{-1} \in A\right) \\
& \rightarrow\left(y^{-1} \in A\right) \\
\vDash_{\lambda}\left(y^{-1} \in A\right) & \rightarrow\left(T\left(1, y^{-1} \in A\right)\right) \\
& \rightarrow\left(T\left((e \in A),\left(y^{-1} \in A\right)\right)\right) \\
& \rightarrow\left(e\left(y^{-1}\right)^{-1} \in A\right) \\
& \rightarrow(e y \in A) \\
& \rightarrow(y \in A)
\end{aligned}
$$

$\vDash_{\lambda}(T((x \in A),(y \in A)))$

$\rightarrow\left(T\left((x \in A),\left(y^{-1} \in A\right)\right)\right)$

$\rightarrow\left(x\left(y^{-1}\right)^{-1} \in A\right)$

$\rightarrow(x y \in A)$

Therefore $A$ is an implication-based T-fuzzy subgroup of $G$.

This serves as the necessary and sufficient condition for a fuzzy subset $A$ of $G$ to be an implication-based T-fuzzy subgroup of $G$.

Theorem 9. Let $A$ be an implication-based T-fuzzy subgroup of $G$ such that $\vDash_{\lambda}(a \in A) \rightarrow 1 . f_{a}: G \rightarrow G$ is called as right translation and is defined as $f_{a}(x)=x a$ and ${ }_{a} f: G \rightarrow G$ is called as left translation and is defined as ${ }_{a} f(x)=a x$. Then $f_{a}(A)={ }_{a} f(A)=A$.

Proof. Let $x \in G$ $\vDash_{\lambda}(x \in A)$ 
$\rightarrow(T((x \in A), 1))$

$\rightarrow(T((x \in A),(a \in A)))$

$\rightarrow\left(T\left((x \in A),\left(a^{-1} \in A\right)\right)\right)$

$\rightarrow\left(x a^{-1} \in A\right)$

$\rightarrow\left(\exists z\{(z \in A)\} ; z \in f_{a}^{-1}(x)\right)$

$\rightarrow\left(x \in f_{a}(A)\right)$

$\vDash_{\lambda}\left(x \in f_{a}(A)\right)$

$\rightarrow\left(x a^{-1} \in A\right)$

$\rightarrow\left(T\left(\left(x a^{-1} \in A\right), 1\right)\right)$

$\rightarrow\left(T\left(\left(x a^{-1} \in A\right),(a \in A)\right)\right)$

$\rightarrow\left(x a^{-1} a \in A\right)$

$\rightarrow(x \in A)$

$\therefore f_{a}(A)=A$.

Similarly we can prove that ${ }_{a} f(A)=A$.

Let $x \in G$

$\vDash_{\lambda}(x \in A)$

$\rightarrow(T(1,(x \in A)))$

$\rightarrow(T((a \in A),(x \in A)))$

$\rightarrow\left(T\left(\left(a^{-1} \in A\right),(x \in A)\right)\right)$

$\rightarrow\left(a^{-1} x \in A\right)$

$\rightarrow\left(\exists z\{(z \in A)\} ; z \in_{a} f^{-1}(x)\right)$

$\rightarrow(x \in a f(A))$

$\vDash_{\lambda}\left(x \in \in_{a} f(A)\right)$

$\rightarrow\left(a^{-1} x \in A\right)$

$\rightarrow\left(T\left(1,\left(a^{-1} x \in A\right)\right)\right)$

$\rightarrow\left(T\left((a \in A),\left(a^{-1} x \in A\right)\right)\right)$

$\rightarrow\left(a a^{-1} x \in A\right)$

$\rightarrow(x \in A)$

$\therefore \quad{ }_{a} f(A)=A$.

Definition 10. Let $A$ and $B$ be two implication-based T-fuzzy subgroups of G. Then the implication-based T-fuzzy product of $A$ and $B$ denoted by $[A \cdot B]_{T}$ is defined as

$\vDash_{\lambda}(\exists y, z\{T((y \in A),(z \in B))\} ; y z=x ; y, z \in G)$ $\rightarrow(x \in A \cdot B) \forall x \in G$.

Definition 11. Let $A$ and $B$ be two implication-based T-fuzzy subgroups of G. Then the implication-based $\dot{T}$-fuzzy product of $A$ and $B$ denoted by $[A \cdot B]_{\dot{T}}$ 
is defined as

$\vDash_{\lambda}(T((x \in A),(x \in B))) \rightarrow\left(x \in[A \cdot B]_{\dot{T}}\right) \forall x \in G$.

Definition 12. Let $G_{1}$ and $G_{2}$ be two finite groups and $G=G_{1} \times G_{2}$ be the direct product group of $G_{1}$ and $G_{2}$. Let $A_{1}$ and $A_{2}$ be two implicationbased T-fuzzy subgroups of $G_{1}$ and $G_{2}$ respectively and $T^{\prime}$ be a $t$-norm. Then the implication-based $T^{\prime}$-fuzzy direct product of $A_{1}$ and $A_{2}$ denoted by $\left[A_{1} \times A_{2}\right]_{T^{\prime}}$ is defined as

$$
\begin{aligned}
& \vDash_{\lambda}\left(T^{\prime}\left(\left(a \in A_{1}\right),\left(b \in A_{2}\right)\right)\right) \rightarrow\left((a, b) \in\left[A_{1} \times A_{2}\right]_{T^{\prime}}\right) \\
& \forall a \in G_{1}, b \in G_{2} .
\end{aligned}
$$

Theorem 10. Let $G_{1}$ and $G_{2}$ be two finite groups and $G=G_{1} \times G_{2}$ be the direct product of $G_{1}$ and $G_{2}$. Let $A_{1}$ and $A_{2}$ be two implication-based T-fuzzy subgroups of $G_{1}$ and $G_{2}$ respectively. Then $A=\left[A_{1} \times A_{2}\right]_{T}$ is an implication-based T-fuzzy subgroup of $G$.

Proof. Let $x=\left(a_{1}, b_{1}\right)$ and $y=\left(a_{2}, b_{2}\right)$ be any two elements of the group $G=G_{1} \times G_{2}$.

$\vDash_{\lambda}(T((x \in A),(y \in A)))$

$\rightarrow\left(T\left(\left(\left(a_{1}, b_{1}\right) \in A\right),\left(\left(a_{2}, b_{2}\right) \in A\right)\right)\right)$

$\rightarrow\left(T\left(T\left(\left(a_{1} \in A_{1}\right),\left(b_{1} \in A_{2}\right)\right)\right.\right.$,

$$
\left.T\left(\left(a_{2} \in A_{1}\right),\left(b_{2} \in A_{2}\right)\right)\right)
$$

$\rightarrow\left(T\left(T\left(\left(a_{1} \in A_{1}\right),\left(a_{2} \in A_{2}\right)\right), T\left(\left(b_{1} \in A_{1}\right),\left(b_{2} \in A_{2}\right)\right)\right)\right.$

by the generalised associative law

$\rightarrow\left(T\left(\left(a_{1} a_{2}{ }^{-1} \in A_{1}\right),\left(b_{1} b_{2}{ }^{-1} \in A_{2}\right)\right)\right)$

$\rightarrow\left(\left(a_{1} a_{2}^{-1}, b_{1} b_{2}^{-1}\right) \in A\right)$

$\rightarrow\left(x y^{-1} \in A\right)$

Let $e=\left(e_{1}, e_{2}\right) \in G$ where $e_{1}, e_{2}$ are the identity elements of the group $G_{1}$ and $G_{2}$ respectively.

$$
\begin{aligned}
& \vDash_{\lambda}\left(T\left(\left(e_{1} \in A_{1}\right),\left(e_{2} \in A_{2}\right)\right)\right) \\
& \\
& \rightarrow\left(\left(e_{1}, e_{2}\right) \in A\right) \\
& \rightarrow(e \in A)
\end{aligned}
$$

$$
\text { But } \begin{aligned}
\vDash_{\lambda}(T( & \left.\left.\left(e_{1} \in A_{1}\right),\left(e_{2} \in A_{2}\right)\right)\right) \\
& \rightarrow(T(1,1)) \\
& \rightarrow 1
\end{aligned}
$$

$$
\therefore \vDash_{\lambda}(e \in A) \rightarrow 1
$$

By theorem $8 A=\left[A_{1} \times A_{2}\right]_{T}$ is an implication-based T-fuzzy subgroup of G. 
Theorem 11. Let $G_{1}$ and $G_{2}$ be two finite groups and $G=G_{1} \times G_{2}$ be the direct product of $G_{1}$ and $G_{2}$. Let $A$ and $B$ be two implication-based $T$ fuzzy subgroups of $G_{1}$ and $G_{2}$ respectively. Then $[A \times B]_{T^{\prime}}$ is an implicationbased $T$-fuzzy subgroup of $G$ provided $T^{\prime}$ dominates $T$. Moreover if $A$ and $B$ are implication-based T-fuzzy normal subgroup of $G$ then $[A \times B]_{T^{\prime}}$ is an implication-based T-fuzzy normal subgroup of $G$.

Proof. Let $x, y \in G$ where $x=\left(a_{1}, b_{1}\right)$ and $y=\left(a_{2}, b_{2}\right) ; a_{1}, a_{2} \in G_{1}$ and $b_{1}, b_{2} \in G_{2}$.

$\vDash_{\lambda}\left(T\left(\left(x \in[A \times B]_{T^{\prime}}\right),\left(y \in[A \times B]_{T^{\prime}}\right)\right)\right)$

$\rightarrow\left(T\left(\left(\left(a_{1}, b_{1}\right) \in[A \times B]_{T^{\prime}}\right),\left(\left(a_{2}, b_{2}\right) \in[A \times B]_{T^{\prime}}\right)\right)\right)$

$\rightarrow\left(T\left(\left(T^{\prime}\left(\left(a_{1} \in A\right),\left(b_{1} \in B\right)\right)\right),\left(T^{\prime}\left(\left(a_{2} \in A\right),\left(b_{2} \in B\right)\right)\right)\right)\right)$

$\rightarrow\left(T^{\prime}\left(\left(T\left(\left(a_{1} \in A\right),\left(a_{2} \in A\right)\right)\right),\left(T\left(\left(b_{1} \in B\right),\left(b_{2} \in B\right)\right)\right)\right)\right)$

$\because T^{\prime}$ dominates $T$

$\rightarrow\left(T^{\prime}\left(\left(a_{1} a_{2}{ }^{-1} \in A\right),\left(\left(b_{1} b_{2}{ }^{-1} \in B\right)\right)\right)\right.$

$\rightarrow\left(\left(a_{1} a_{2}{ }^{-1}, b_{1} b_{2}{ }^{-1}\right) \in[A \times B]_{T^{\prime}}\right)$

$\rightarrow\left(x y^{-1} \in[A \times B]_{T^{\prime}}\right)$

Let $e=\left(e_{1}, e_{2}\right) \in G$

$\vDash_{\lambda}\left(T^{\prime}\left(\left(e_{1} \in A\right),\left(e_{2} \in B\right)\right)\right)$

$\rightarrow\left(\left(e_{1}, e_{2}\right) \in[A \times B]_{T^{\prime}}\right)$

$\rightarrow\left(e \in[A \times B]_{T^{\prime}}\right)$

But $\vDash_{\lambda}\left(T^{\prime}\left(\left(e_{1} \in A\right),\left(e_{2} \in B\right)\right)\right)$

$\rightarrow\left(T^{\prime}(1,1) \in[A \times B]_{T^{\prime}}\right)$

$\rightarrow 1$

$\therefore \vDash_{\lambda}\left(e \in[A \times B]_{T^{\prime}}\right) \rightarrow 1$

By theorem 8

$[A \times B]_{T^{\prime}}$ is an implication-based T-fuzzy subgroup of $\mathrm{G}$.

$\vDash_{\lambda}\left(T^{\prime}((x y \in A),(x y \in B))\right)$

$\rightarrow\left(x y \in[A \times B]_{T^{\prime}}\right)$

But $\vDash_{\lambda}\left(T^{\prime}((x y \in A),(x y \in B))\right)$

$\rightarrow\left(T^{\prime}((y x \in A),(y x \in B))\right)$

$\because \mathrm{A}$ and $\mathrm{B}$ are implication-basedT-fuzzy normal subgroups of $\mathrm{G}$

$\rightarrow\left(y x \in[A \times B]_{T^{\prime}}\right)$

$[A \times B]_{T^{\prime}}$ is an implication-based T-fuzzy normal subgroup of G.

Theorem 12. Let $i^{2}: G \rightarrow G \times G$ defined by $i^{2}(x)=(x, x) \forall x \in G$. If $A$ and $B$ are two implication-based T-fuzzy subgroups of $G$ then $[A \cdot B]_{\dot{T}}$ is the pre-image of $[A \times B]_{T}$ under $i^{2}$.

Proof. Let $x \in G$. By definition we have 
$\vDash_{\lambda}(T((x \in A),(x \in B))) \rightarrow\left((x, x) \in[A \times B]_{T}\right)$

$\vDash_{\lambda}(T((x \in A),(x \in B))) \rightarrow\left(x \in[A \cdot B]_{\dot{T}}\right)$

$\Rightarrow \quad \vDash_{\lambda}\left((x, x) \in[A \times B]_{T}\right) \rightarrow\left(x \in[A \cdot B]_{\dot{T}}\right)$

$\Rightarrow \vDash_{\lambda}\left(i^{2}(x) \in[A \times B]_{T}\right) \rightarrow\left(x \in[A \cdot B]_{\dot{T}}\right)$

$\therefore \quad[A \cdot B]_{\dot{T}}$ is the pre-image of $[A \times B]_{T}$ under $i^{2}$.

Theorem 13. Let $A$ and $B$ be two implication-based T-fuzzy normal subgroups of a group G. Let $T^{\prime}$ be a t-norm which dominates T. Then $[A \cdot B]_{\dot{T}^{\prime}}$ is an implication-based T-fuzzy normal subgroup of $G$.

Proof. $\vDash_{\lambda}\left(T^{\prime}((e \in A),(e \in B))\right) \rightarrow\left(e \in[A \cdot B]_{\dot{T}^{\prime}}\right)$

But $\vDash_{\lambda}\left(T^{\prime}((e \in A),(e \in B))\right) \rightarrow\left(T^{\prime}(1,1)\right) \rightarrow 1$

$\therefore \vDash_{\lambda}\left(e \in[A \cdot B]_{\dot{T}^{\prime}}\right) \rightarrow 1$

Let $x, y \in G$

$\vDash_{\lambda}\left(T\left(\left(x \in[A \cdot B]_{\dot{T}^{\prime}}\right),\left(y \in[A . B]_{\dot{T}^{\prime}}\right)\right)\right)$

$\rightarrow\left(\left(T\left(T^{\prime}((x \in A),(x \in B)), T^{\prime}((y \in A),(y \in B))\right)\right)\right.$

$\rightarrow\left(\left(T^{\prime}(T((x \in A),(y \in A)), T((x \in B),(y \in B)))\right)\right.$

$\because T^{\prime}$ dominates $\mathrm{T}$

$\rightarrow\left(T^{\prime}\left(\left(x y^{-1} \in A\right),\left(x y^{-1} \in B\right)\right)\right)$

$\rightarrow\left(x y^{-1} \in[A \cdot B]_{\dot{T}^{\prime}}\right)$

By theorem $8[A \cdot B]_{\dot{T}^{\prime}}$ is an implication-based T-fuzzy subgroup of G.

$\vDash_{\lambda}\left(T^{\prime}((x y \in A),(x y \in B))\right) \rightarrow\left(x y \in[A \cdot B]_{\dot{T}^{\prime}}\right)$

But

$\vDash_{\lambda}\left(T^{\prime}((x y \in A),(x y \in B))\right) \rightarrow\left(T^{\prime}((y x \in A),(y x \in B))\right)$

$\because \mathrm{A}$ and $\mathrm{B}$ are implication-based T-fuzzy normal subgroups of $\mathrm{G}$

$\rightarrow\left(y x \in[A \cdot B]_{\dot{T}^{\prime}}\right)$

$[A \cdot B]_{\dot{T}^{\prime}}$ is an implication-based T-fuzzy normal subgroup of $\mathrm{G}$.

We have if $f$ is an homomorphism on a group $\mathrm{G}$ and $\mathrm{A}$ and $\mathrm{B}$ are implicationbased T-fuzzy subgroups of $f(G)$. If the t-norm $T^{\prime}$ dominates T. Then $[A \cdot B]_{\dot{T}^{\prime}}$ is an implication-based T-fuzzy subgroup of $f(G)$. By theorem $3 f \circ A, f \circ B$ and $f \circ[A \cdot B]_{\dot{T}^{\prime}}$ are implication-based T-fuzzy subgroups of $G$.

Theorem 14. Let $f$ be an homomorphism on a finite group $G$. Let $B_{1}$ and $B_{2}$ be two implication-based $T$-fuzzy subgroups of $f(G)$. Let the t-norm $T^{\prime}$ dominate $T$. If $B=\left[B_{1} \cdot B_{2}\right]_{\dot{T}^{\prime}}$ is the implication-based $\dot{T}$-fuzzy product of $B_{1}$ and $B_{2}$ and $\left[A_{1} \cdot A_{2}\right]_{\dot{T}^{\prime}}$ is the implication-based $\dot{T}$-fuzzy product of $A_{1}=f \circ B_{1}$ and $A_{2}=f \circ B_{2}$ then

$\vDash_{\lambda}\left(x \in\left[A_{1} \cdot A_{2}\right]_{\dot{T}^{\prime}}\right) \rightarrow(x \in A) \quad \forall x \in G$ where $A=f \circ B$. 


\author{
Proof. Let $x \in G$
}

But

$$
\vDash_{\lambda}\left(T^{\prime}\left(\left(x \in A_{1}\right),\left(x \in A_{2}\right)\right)\right) \rightarrow\left(x \in\left[A_{1} \cdot A_{2}\right]_{\dot{T}^{\prime}}\right)
$$

$$
\begin{aligned}
\vDash_{\lambda}\left(T^{\prime}\right. & \left.\left(\left(x \in A_{1}\right),\left(x \in A_{2}\right)\right)\right) \\
& \rightarrow\left(T^{\prime}\left(\left(f(x) \in B_{1}\right),\left(f(x) \in B_{2}\right)\right)\right) \\
& \rightarrow\left(f(x) \in\left[B_{1} \cdot B_{2}\right]_{\dot{T}^{\prime}}\right) \\
& \rightarrow(f(x) \in B) \\
& \rightarrow(x \in A)
\end{aligned}
$$

\title{
References
}

[1] Zadeh L.A,Fuzzy Sets,Information Control,8, (1965), 338-353. [doi: 10.1016/S00199958(65)90241-X]

[2] Azriel Rosenfeld, Fuzzy Groups, Journal of Mathematical Analysis and applications, 35, (1971), 512-517. [doi: 10.1016/0022-247X(71)90199-5]

[3] Dib K.A and Hassan A.A ,The Fuzzy Normal Subgroup,Fuzzy sets and systems, 98, (1998), 393-402. [doi: 10.1016/S0165-0114(96)00338-7]

[4] Malik D.S,John N. Mordeson, and P.S. Nair, Fuzzy Normal Subgroups in Fuzzy Subgroups, J. Korean Math. Soc., 29, (1992), 1-8.

[5] Mukherjee N.P and Prabir Bhattacharya, Fuzzy Normal Subgroups and Fuzzy Cosets, Information Sciences, 34, (1984), 225 - 239. [doi: 10.1016/0020-0255(84)90050-1]

[6] Anthony J.M. and Sherwood H., Fuzzy Groups Redefined, J. Math. Anal. Appl., 69, (1979), 124 - 130. [doi: 10.1016/0022-247X(79)90182-3]

[7] Salvatore Sessa, On Fuzzy Subgroups and Fuzzy Ideals under trianglular norm, Fuzzy Sets and System, 13, (1984), 95-100. [doi: 10.1016/0165-0114(84)90028-9]

[8] Xuehai Yuan, Cheng Zhang, Yonghong Ren,Generalized fuzzy groups and manyvalued implications, Fuzzy Sets and System, 138, (2003), 205-211. [doi: 10.1016/S01650114(02)00443-8]

[9] Selva Rathi. M and Michael Anna Spinneli.J, Implication-Based Fuzzy Normal Subgroup of a Finite group, International Journal of Applied Engineering Research, Vol 10, No. 80, (2015), 5 - 8 .

[10] Abu Osman M.T, On the Direct Product of Fuzzy Subgroups, Fuzzy Sets and Systems, 12, (1984), 87 - 91. [doi: 10.1016/0165-0114(84)90052-6]

[11] Abu Osman M.T, On Some Product of Fuzzy Subgroups, Fuzzy Sets and Systems, 24, (1987), 79 - 86. [doi: 10.1016/0165-0114(87)90115-1] 
